\title{
The use of the fastrak system to evaluate the independent movements of ankle, subtalar and midtarsal joints during walking.
}

\author{
${ }^{1}$ Dr Sayaji V Bhamre, ${ }^{2}$ Dr Rami J Abboud, ${ }^{3}$ Mr Graham Arnold. \\ ${ }^{1,2,3}$ Tort centre, Ninewells Hospital and medical school, Dnundee, Scotland
}

\section{Introduction}

Healthy humans walk with bipedal gait, which is a coordinated series of movements occurring through the body and, in lower limb. The ankle and foot play an important role in gait cycle. It is necessary to study biomechanics of the ankle and foot as it provides powerful tools in studying both normal and pathological gait, for example cerebral palsy, neuromuscular disorders, and traumatic conditions like ankle injuries(Scott and winter,1991). In normal gait the lower limb undergoes various complex mechanism, but the significant mechanism of foot function can be explained by studying the biomechanics of three particular joints; the ankle, the subtalar and midtarsal joints(Bevan, 1992). Movements of the ankle, subtalar and midtarsal joints during normal walking have not been studied independetly until now.

The ankle and foot undergo a very complex mechanism of various movements during the gait cycle. This take place about joint axes, which occur in one, two, or three body planesat a time. with conventional method it is not possible to measure joint movements in all three planes at a time. Method such as three dimensional video(Vicon system) gait analysis are both time consuming and expensive.

The Fastrak system is an electromagnetic device which determines the position and orientation of an object in space three dimensionally. With the fastrak system it is possible to calculate three palmer movements of the joints. Compare to other devices the Fastrak system is inexpensive, reliable, less time consuming and proved to be repeatable (Rendall and Abboud, 1999).

\section{Aims and Objectives:}

Aim:

To use the fastrak system to evaluate the independent movements of ankle, subtalar and midtarsal joints during walking.

\section{Objectives:}

1) Identify the environment.

2) Devise experimental procedure, protocol and methodology.

3) Develop a protocol to identify data for each of the three respective joints.

4) Establish normal range of movements for each joints during normal walking.

\section{Method And Material:}

This study was conducted at the institute of Motion Analysis and research (IMAR) in the Tayside Orthopaedic and rehebilitation Technology (TORT) Centre, Ninewells Hospital and Medical School, University of Dundee, Scotland. The collection of data commenced in February 2006 and reached completion in April 2006.

Healthy volunteers recruited from displaced posters in the university of Dundee and Ninewells Hospital. Age of the volunteers varied from 18 to 65 years. voiunteers wee excluded from the study if they had experienced any foot pain within last six months, any past history of lower limb surgery, any congenital or acquired deformities of the feet.

\section{Adaptation of the system for the use on the ankle-foot:}

The Fastrak system: The fastrak system can be used to measure the position of an object in space. The system employs a motion capture unit (MCU), which contains the circuitry; hardware and software to control digitise the elctromagnetic signals. The trasmitter, which contains three mutually orthogonal coils, generates three different electromagnetic fields in $30 \mathrm{cubic} \mathrm{cm}$ region. Sensor also containing three coils, records magnetic flux in the three different fields. Magnetic flux generates propotional currents used to calculate a vector, which signifies the direction and strength of the magnetic field at the site of the sensor. Dedicated software computes the position ( $\mathrm{x}, \mathrm{y}, \mathrm{z}$ cartesian coordinates) and orientation (azimuth, elevation and roll) of the tracking sensor. 


\section{The GAITRite System:}

In this study the Fastrak system was synchronised with the GAITRite system to record the ankle/foot joint movements at various stages during the stance phase of the gait cycle. The GAITRite system automates the measuring of temporal (timing) and spatial ( distance) gait parameters via an electronic walkway connected to the serial port of a personal computer. The standard GAITRite electronic walkway contains 6 sensor pads enclosed in a roll-up carpet which has an active area of 24 inches in width and 144 incehes in lenght. The Fastrak system was synchronised with the GAITRite system by a spwcially designed synhronization box at IMAR, Dundee. this box adjusted the frequency of the Fastrak system $(40 \mathrm{~Hz})$ to recognize the instant of heelstrie and toeoff using temporal gait parameters obtained from GAITRite system.

\section{Identification of the environment:}

The Fastrak system is an electromagnetic device. magnetic fields are generated from a trasmitter with three coils, which are placed orthogonally. Any metal object within this field between sensor and trasmitter leads to errore in the angle recording. All the metel objects situiated within furniture, electric device and power lines were kept waway from the area of experiment. The mounting device was constructed from orthopaedic plastic. plastic bolts and screws were used to fix the transmitter to the the brace instead of metal screws.

\section{Calibration and Orientation of the Fastrak system:}

The system developed by Rendall And Abboud (1999) was used to align the trasmitter with the three cardinal planes of the body so movement of the receiver could be recorded as anatomical movement of that particular joint. A wooden platform $(0.36 \mathrm{sq} . \mathrm{m})$ with a vertical centreboard was constructed. the platform constitutes a static reference frame representing three planes (side and bace) perpendicular to each other. The receiver was fixed to the stsatic reference frame in parallel to the three sides using th etrasmitter signal as a reference. It was confirmed that the receiver was parallel to the three cardinal planes by adjusting its position accordingly. A small lightweight brace was constructed with rigid orthopaedic plastic in such a way that it could fit closely over the tibia shin, thus avoiding any unnecessary movement between the brace and the underlying bone. This brace was lined with sysnthetic rubber, which gave greater structural firmness to the brace when mounted on the leg. The trasmitter was fixed to the brace in a locking ball and socket joint to permit both free orientation of the transmitter to align it with anatomical planes and fix it in desired position once it was aligned with anatomical palnes of the body. Aligning the trasmitter with anatomical planes of the body ensured that the movement of the sensor relative to the transmitter could be an anatomical movement of calcaneum and navicular relative to tibia in frontal, sagittal and transverse planes. The trasmitter was removed from the frame and fixed over the leg of the subject with a brace. The subject was then asked to stand on the platform with one leg placed on either side of the centre board in such way that their weight was evenly distributed on both feet. The trasmitter was aligned with the help of a ball and socket joint parallel to the three cardinal planes of the body by using the transmitter and receiver signals. The trasmitter was adjusted until it matched the reading that were taken at the time of initial adjustment on the wooden platform. This confirmed that the transmitter was paralllel to the sagittal plane of the tibia and movements of the desired joints would be recorded in relation to the sagaittal plane of the tibia.

It was decided to position the transmitter over the front of lower third of tibia, $10 \mathrm{~cm}$ proximal to the tip of medial malleolus reason being, it reduced the distance between transmitter and the reiceiver, which in turn reduced the potential for error occuring when data recording. Secondly, as the front of the tibia has little subcutaneous tissue which reduces possible skin movement relative to the underlying bone. The subject was asked to walk normally in gait laboratory, while data was recorded to see any relative movement of the transmitter. The data was analysed to see if any movement of the transmitter occurred in the three panes. there was absolutely no movement in eitherthe frontal and sagittal planes $\left(\leq 0.25^{\circ}\right)$ and the transverse plane transmitter showed movement less than $2^{\circ}$ which was acceptable.

It was decide to attach one sensor over the lateral aspect of the calcaneum and another over the navicular tubercle in order to record the subtalar and midtarsal joint movement respectivley. a plastic mould was used as a stable platform to ensure that the sensor was in close contact with the skin surface, thus avoiding any unnecessary movement of the receiver locally, especially rotation. both sensors were attached to the foot with double-sided adhesive tape.

\section{Protocol for the assessments of ankle, subtalar and midtarsal movements:}

Once the method for attachment of the transmitter and sensor had been agreed upon, a protocol for the set up and analysis of the joint movements were considered. Once sensors and trasmitter were attached to the subject, the subject was then asked to to stand, with knees extended, looking straight ahead, and applying equal weight on both legs. Particular care was taken while the subtalar joint was in neutral position that the foot and ankle formed a $90^{\circ}$ angle. At this stage a software-drivenboresight or neutral orientation procedure was 
performed for both sensors. The boresight aligned the sensor position to the transmitter as zero in all three planes. The boresight position of the sensor and transmitter was used as a reference frame to calculate the angles of the ankle, subtalar and midtarsal joints during walking. Once the reference frame was set up, subject was asked to walk on the GAITRite walkway to recored spatial(time) parameters while Fastrak syatem recorded the angles during walking. The Fastrak system started recording the data as soon as the subject started walking on the GAITRite walkway.

The spatial(time) parameters obtained from the GAITRite data were used to find out the timing of heelstrike and toe-off, so accordingly the relative change in particular joint angles waas calculated from the fastrak data.

The angles received from the Fastrak system wer euler angles. According to Euler's rotation theoram, any rotation may be described using three angles. These three angles are azimuth, elevation and roll/tilt. If Angles measured in Euler angles with fastrak system, any change in position of the receiver in any one plane or two will give change in position of the receiver in third plane though there were no change in position in third plane. In This study we were interested in calculating absolute angles around particular axes to find the joint movements around that particular axis. This was possible only when the angles were calculated in axis angles. The conversion of the euer angle to cartesian angle gave the actual change in the angle of the joint around that particular axis. To convert the euler angle to an axis angle, a custom-made computer software developed at IMAR was used. This ensured that the movement recorded in the foot were about a particular Cartesian axis.

Data was collected from Fastrak And GAITRite syatem. The data from both systems was then synchronized with specially designed computer software. The combined data from both system was opened in a Microsoft Excel file. Reiceiver one represented the movements of the midtarsal joint and receiver two represented the movements of the ankle and subtalar joints. The data was extracted for the adduction/abduction, planterflesion/dorsiflexion and inversion/eversion movements at hindfoot and forefoot. These combined files were studied to extract the various variables. The data was extracted for the stance phase of the gait cycle. These variables were ankle, subtalar and midtarsal movements at heel strike, midstance and toe off. The adduction/abduction, planterflexion/dorsiflexion and inversion/eversion represented the movements in the transverse, sagittal and frontal planes respectively for both joints. This data was analysed clinically as well as statistically.

\section{Statistical analysis:}

SPSS software was used to statistically analyse the data. Data was obtained from the same subject on two occasions, on two different days with an interval of two weeks( 15 subjects) and one week (5 subjects). The Data was analysed for 40 feet. Descriptive analysis was carried out to discover the mean values of each variable including its standard deviation. The Shapiro-wilk test was carried out to test the normality of the distribution of the values around the mean. The maximum and minimum angles at particular joint position were calculated. Histograms wer plotted for each variable and were tested for normality by the Shapiro-wilk test.

\section{Results:}

The movements of the ankle, subtalar and midtarsal joints described in this study were always referred to in the anatomical position. The dorsiflexion/planterflexion occured in the sagittal plane, inversion/eversion occured in the frontal plane and adduction/abduction occured in the transverse plane.

Twenty volunteers were recruited from the displayed posters at various locations throughout the university of Dundee main campus. The study had been reviewed and approved by the university research ethics subcommittee. Of the 20 volunteers, 3 were females and 17 were male, all data was collected for the right foot only.

The synchronised data files of the Fastrak and GAITRite systems were opened in Microsoft Excel software. The GAITRite data was used to find out the instant of heel strike, midstance and toe off of both feet, and the Fastrak data provided the particular angles at a particular instant during stance phace of the gait cycle for the right foot. The middle reading between the heel strike(first contact) and toe off(last contact) Of a right foot was taken as the midstance. The data was identified for second step rather than first step an dthird steps of the walk thus avoiding potential problems with acceleration and deceleration at the end of the walk. This ensured that the data coleected fro the step two gave as normal angles as realistically can be acheived while walking. The data was identified for three walks of the same individual on two different days. Graphs were also plotted in Microsoft Excel to correlate the movements of all three joints during the gait cycle to see any graph pattern. The positive angles indicated th planterflexion, inversion, and adduction and negative angles indicated the dorsiflexion eversion and abduction of ankle, subtalar and midtarsal joints. The graphical representation for forefoot and hindfoot movement is as follows- 

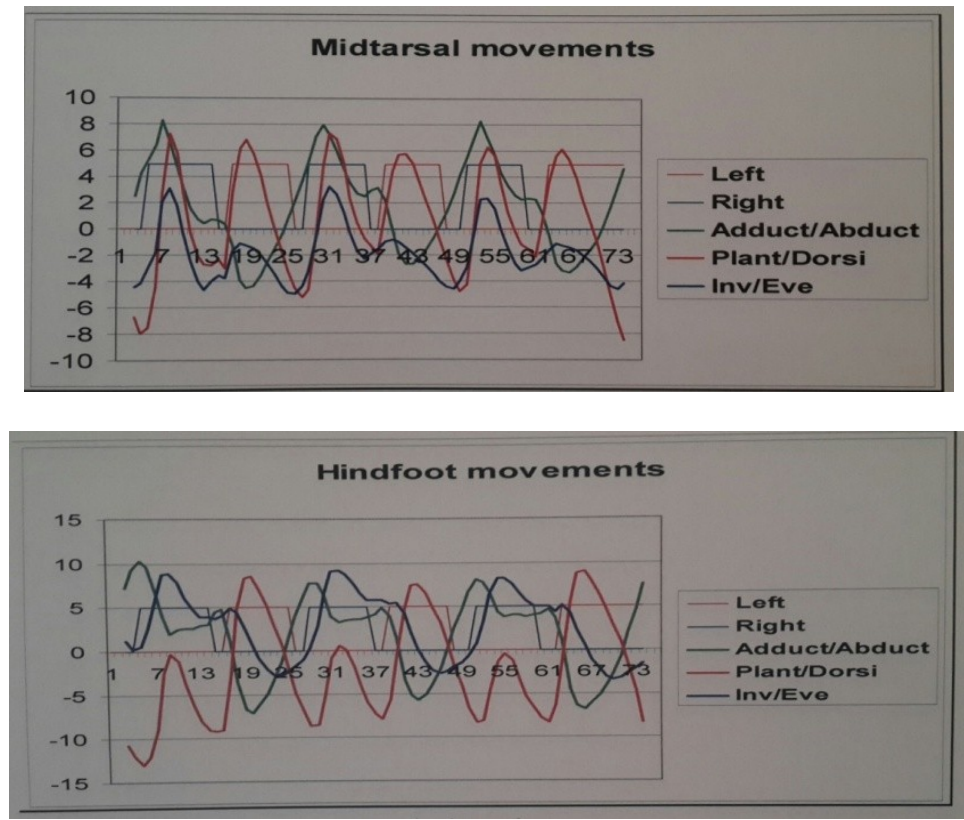

V. Movements of the ankle, Subtalar and midtarsal joints:

\section{Movements of ankle joint in sagittal plane(planterflexion/dorsiflexion):}

The ankle joint was slightly dorsiflexed before heel cotact. After the heel made contact with the ground the ankle joint rapidly planterflexed to a maximum just before foot flat. the mean plantarflexion at heel contact was $1.7^{\circ}$ and standard deviation was 4.1. The maximum planterflexion after heel contact was $13.07^{\circ}$ and minimum was $-7.69^{\circ}$. The rear foot then dorsiflexed progressively until it reached the maximum dorsiflexion after midstance. The mean angle of dorsiflexion at midstance was $0.31^{\circ}$ (SD 3.30). The maximum/minimum range for dorsiflesion was $-9.74^{\circ}$ to $10.21^{\circ}$.

Rapid planterflexion occured just after the midstance until toe off. The mean angle at toe off for planterflexion was $-3.06^{\circ}$ (SD 3.23). The range for maximum/minimum planterflexion at toe off was $4.79^{\circ}$ to $9.69^{\circ}$.

\section{Movements of subtalar joint in frontal plane(inversion/eversion):}

At heel contact the rear foot was slightly inverted with a mean value of $4.3^{\circ}$ (SD 2.1). The maximum/minimum range for inversion at heel strike was from $9.75^{\circ}$ to $-0.45^{\circ}$. Evesion gradually occured from heelstrike to midstance of the gait cycle. The mean eversion at midstance was $-1.12^{\circ}$ (SD 2.7). The maximum/minimum range for eversion at midstance was $-5.86^{\circ}$ to $12.19^{\circ}$. The subtalar joint then progressively inverted until toe off. The mean inversion at toe off was $13.24^{\circ}$ to $-1.24^{\circ}$.

\section{Movements of the hind-foot in transverse plane (abduction/adduction):}

At the stance phace of the gait cycle, as the foot is flexed to the ground, tibial rotation was considered to calculate hindfoot and forefoot abduction and adduction. At heel strike the rear foot was in slight abduction. The mean abduction at heel contact was $5.71^{\circ}$ to $-7.49^{\circ}$. Abduction of the rear foot increased from heel contact prior to the midstance (foot flat). Then the rear foot adducted from just before midstance to toe off progressively. The mean abduction/adduction at midstance was $-2.6^{\circ}$ (SD 2.70). The maximum/minimum range of abduction at midstance was $-9.59^{\circ}$ to $4.02^{\circ}$. From foot flat throuh midstance, the rear foot reached maximum adduction at toe off. The mean adduction at toe off was $2.4^{\circ}$ (SD 2.45). The maximum/minimum range was from $7.47^{\circ}$ to $-3.73^{\circ}$.

\section{Movements of the midtarsal joint in sagittal plane (planterflexion/dorsiflexion):}

The midtarsal joint was slightly dorsiflexed prior to heel contact. After heel contact the midtarsal joint rapidly planterflexed to a maximum just before footflat. The mean planterflexion at heel contact was $2.22^{\circ}$ (SD 4.08). The maximum/minimum range for planterflexion at heel contact was from $10.58^{\circ}$ to $-13.31^{\circ}$. The midtarsal joint then dorsiflexed progressively, reaching the maximum after midstance. The mean angle for doesiflexion at midstance was $-0.21^{\circ}$ (SD 3.35). The max/min range for dorsiflesion at midstance was $-14.60^{\circ}$ to $8.54^{\circ}$.

Rapid plantarflexion occured just after the midstance until toe off. The mean angle at toe off for plantarflexion for the midtarsal joint was $-0.95^{\circ}$ (SD 3.97). The max/min range for planterflexion at toe off was from $11.86^{\circ}$ to $-9.13^{\circ}$. 


\section{Movements at the midtarsal joint in frontal plane (inversion/eversion):}

The midtarsal joint was slightly inverted at heel contact with the mean value being 1.55 (SD3.83). The $\max /$ min range for inversion at heel contact was from $10.40^{\circ}$ to $-7.10^{\circ}$. Eversion gradually occured from heelstrike to midstance of the gait cycle. Mean eversion at midstance was from $-10.64^{\circ}$ to $12.83^{\circ}$. The subtalar joint then progressivley inverted until toe off. The mean inversion at tor off was $-0.43^{\circ}$ (SD 3.9). The $\mathrm{max} / \mathrm{min}$ range for inversion at toe off was from $8.95^{\circ}$ to $-9.55^{\circ}$.

\section{Movements of the midtarsal joint in transverse plane(abduction/adduction):}

At the stance phase of the gait cycle, as the foot is fixed to the ground, tibial rotation was considered to calculate forefoot abduction and adducton. The forefoot was in slight abduction at heel strike. The mean abduction at heel contact was $0.75^{\circ}$ (SD 2.8). The max/min range for abduction at heel contact was from6.28 to $-7.2^{\circ}$. Abduction of the forefoot increased from heel contact to prior to midstance(foot flat). The forefoot then adducted from just prior midstance to toe off prograssively. The mean abduction at midstance was $-2.44^{\circ}$ (SD 2.87). The $\mathrm{max} / \mathrm{min}$ range was $-9.74^{\circ}$ to $8.08^{\circ}$. From foot flat through midstance the forefoot reached its maximum adduction at toe off. The meanadduction at toe off was $3.61^{\circ}$ (SD 3.39). THE max/min range was $9.83^{\circ}$ to $-4.52^{\circ}$

\section{Table A: Test of Normality}

\section{Shapiro-Wilk Teat}

Hindfoot adduction/abduction at heel strike ankle plantar/dorsiflexion at heel strike subtalar inversion/eversion at heel strike Hindfoot addction/abduction at midstance ankle plantar/dorsiflexion at midstance subtalar inversion/eversion at midstance Hindfoot adduction/abduction at toe off ankle plantar/dorsiflexion at toe off subtalar inversion/eversion at toe off midtarsal addction/abduction at heel strike midetarsal plantar/dorsiflexion at heel strike midtarsal inversion/eversion at heel strike midtarsal addction/abduction at midstance midtarsal planter/dorsiflexion at midstance midtarsal inversion/eversion at midsatnce midtarsal addction/abduction at toe off midtarsal planter/dorsiflexion at toeoff midtarsal inversion/eversion at toe off

All the variables were distributed normally except subtalar inversion/eversion at midstance,hindfoot addction/abduction at toe off, midtarsal adduction/abduction at midstance, midtarsal inversion/eversion at midsatnce, and midtarsal add/abd at toe off $(p$ value $<0.05)$
Significance level

0.23

0.55

0.231

0.444

0.319

0

0.055

0.131

0.331

0.094

0.211

0.078

0.02

0.633

0

0.024

0.995

0.464

The Results of the present study wer compared with the results of previous normal gait studies. The data were compared with other three dimensional gait studies. These studies were normal gait studies carried out with an electromagnetic tracking device and the vicon system (Woodburn et al., 1996).

\section{Discussion:}

The Fastrak system is an electromagnetic tracking device that can be adapted to identify the position of an object in space three dimensionally. It is possible with the fastrak system to measure angles in three dimensions as compared to the standard two arm goniometer, which measures in one plane only. The Fastrak system makes it possible to measure the joint angles on awalking subject. Conventional gonimeters can also be associated with observer error, instrument errors and results can vary from person to person (Milgrom et al., 1985).

Such errors associated with conventional goniometer are avoided by employing the Fastrak system to collect the data and processing this data by computer. another problem stated by Milgrom et al.(1985) was calculating a reference point with which to calculate the angles. This problem is also avoided by using the Fastrak system, firstly, as the transmitter was aligned with anatomical plane of the body, so the angles were 
calculated in relation to the cardinal planes of the body, secondly as for all the subjects the reference point was an anatomical position where the subject was asked to stand looking straight ahead to avoid any movement at ankle and knees, with both knees extended, equal weight on both feet without any eversion or inversion at the heel. This position was taken as the reference position to calculate the angles. This procedure was followed very strictly in order to avoid errors when measuring the angles.

The results with initial data and explored data obtained in this study are comparable with normal gait studies conducted with electromagnetic tracking system and the Vicon system. However some differences were found in certain variables. These may be due to the different methodology followed in different normal three dimensional gait studies. There were shift of the graphs on negative or positive sides of the reference line. This shift of the graps is not normal. As subject walks there should be either positive or negative deviation of the graph from reference line for particular movement. The shift of graph on either positive or negative side could be interpreted as the movement occured in particular direction only, for example, shift of the graph of inversion/eversion movement on positive side. This could be interpreted as either healthy subject was walking with inverted foor throughout the stance phase or there was an eversion but due to the shift of the graph it was not possible to document it in a proper way. The shift of the graph was observed for the movements in either one or two planes.

There could be various possible reasons to cause the shift of the graphs. Firstly, the error might have occured at the time of alignment of the foot to anatomical reference position. Secondly, the error might have occured at boresight function of the software. Thirdly, there might be a possibility of the skin movement underneath the receiver. Fourthly, the wires of the receiver might have restricted the free movements of the receiver along with underlying bone after certain point.

As a comparison to the Vicon system the Fastrak system was found to be more predictable, more cost effective and less time consuming(Ahmed 2005). Rendall and Abboud(1999) used the Isotrak sysytem to demonstrate the repeatability of the system to within $2^{\circ}$. The system $w s=a s$ found to be less time consuming, more reliable and less expensive. However the research must be continued in future to investigate the problem encountered with shift of the graph in relation to the zero reference position. This study shows that The Fastrak system could be used fro clinical evaluation of ankle subtalar and midtarsal joint movements seperately during walking. There are some deficiencies that need to be addressed however. Skin movement is a definite source of error( Rendall and Abboud,1999) and is difficult problem to be resolved.

To conclude, The fastrak system can be a very useful tool in measuring the independent movements of the ankle, subtalar and midtarsal joints during walking provided that the problem encountered with reference position is solved.

\section{References}

[1]. Abboud RJ, Agarawal SK, et al. Adirect method for quantitative measurement of ankle proprioception. The Foot; 9:27-30,1999

[2]. Ahmed SA. assesement and comparision of shoulder movements using the fastrak and vicon system. MCh degree thesis;University of dundee, 2005

[3]. Bevan JS. Biomechanics: A review of foot function in gait. The Foot; 2:79-82,1992

[4]. Lundberg A,Svensson OK, Bylund C. Kinematcs of the foot/ankle complex-part2: pronation and supination. Foot and Ankle: 9(5):248-253,1989

[5]. Milgrom C, Giladi M, Simkin A. The normal range of subtalar inversion and evesion in young males as measured by three different techniques. Foot And Anle;6(3):143-145,1985

[6]. Morris JM. Biomechanics of the foot and ankle. Clinical orhtopaedics and related research; 122: 10-17,1977

[7]. Rendall GC and Abbout RJ. The development of a three plane system for the masurement of the ankle/subtalar joint complex in gait. The Foot; 9:31-39,1999

[8]. Scott SH and Winter DA. Talocrural and talocalcaneal joint kinematics and kinetics during the stance phase of walking. J. Biomechanics; 24(8):743-752,1991

[9]. Woodburn J, Helliwell PS, Barker S. A preliminary study determining the feasibility of elcromagnetic tracking for kinematics at the ankle joint complex. Rheumatology; 38:1260-1268, 1999 www.euclidenspace.com 